\title{
Diagnóstico sistémico-cibernético de una institución de educación superior universitaria
}

\section{Systemic-cybernetic diagnosis of a university higher education institution}

\author{
Robensoy Marco Taipe Castro \\ Facultad de Ingeniería de Sistemas, Universidad Nacional del Centro del Perú (UNCP) Huancayo-Perú. \\ rtaipec@gmail.com
}

Resumen - La investigación incurre en el diagnóstico organizacional mediante la cibernética organizacional y el enfoque sistémico; justificado en la posibilidad de evidenciar la efectividad organizacional, acrecentar conceptos de patologías y arquetipos organizacionales. El objetivo es diagnosticar el sistema de gestión para evidenciar la viabilidad y eficacia del servicio profesional en la institución de educación superior pública. Del mismo modo, la investigación inicia un análisis de patologías y arquetipos sistémicocibernéticos organizacionales, luego, el diagnóstico de las funciones sistémicas plasmados en un modelo de sistema viable de la institución. En síntesis, evidencia las condiciones actuales de la institución de educación superior universitaria desde el enfoque sistémico-cibernético, el sistema de gestión de la calidad ISO 9001:2015 y el modelo de acreditación SINEACE.

Palabras clave: Pensamiento sistémico, Cibernética organizacional, Diagnóstico organizacional, Institución de educación superior universitaria, Sistema de gestión.

Abstract - The research affects organizational diagnosis through organizational cybernetics and the systemic approach; justified in the possibility of evidencing organizational effectiveness, increasing concepts of pathologies and organizational archetypes. The objective is to diagnose the management system to demonstrate the viability and effectiveness of the professional service in the public higher education institution. Similarly, the investigation begins an analysis of pathologies and organizational systemic-cybernetic archetypes, then the diagnosis of systemic functions embodied in a viable system model of the institution. In summary, it evidences the current conditions of the university higher education institution from the systemic-cybernetic approach, the quality management system ISO 9001:2015 and the accreditation model of SINEACE.

Key words: Systems thinking, Organizational cybernetics, Organizational diagnosis, Institution of university higher education, Management system.

\section{Introducción}

La percepción del estudiante en relación a la calidad de la universidad peruana es: "el 55,83\% es mala, el 23,35\% regular, el $2,16 \%$ buena, y el $16,67 \%$ excelente" [1]; en tal razón, si la característica del servicio de educación superior es consecuencia de su sistema organizativo, ¿cuál es el sistema organizativo institucional que genera dicha percepción?

La Universidad es una "institución educativa, parte de un modelo sociopolítico definido, fue fundada para lograr fines sociopolíticos cuyas funciones son: la Formación Profesional, la Investigación y el Servicio social, mal llamada Proyección social” [2]. Sin embargo, "en la práctica la universidad fue reducida a lo puramente académico y dentro de esto a lo profesional; relegando a un segundo plano la investigación y a un tercer lugar la llamada Proyección social" [2].

En el cumplimiento legal y formal por una institución de educación superior está la implantación del "sistema de gestión" basada en procesos, el cual es "la manera particular de la institución en realizar sus actividades para el logro de su fin, algunas son eficientes y otras no".

Por otro lado, el sistema organizativo de la Facultad de Ingeniería Metalúrgica y de Materiales (FIMM), es un 
medio para el cumplimiento de los fines y propósitos de la Facultad. Durante su vida institucional sus integrantes y grupos de interés lo han transformado y continuamente construido, ello ha originado una estructura organizativa que no ha sido claramente puesta de manifiesto. Dicho ocultamiento dificulta el plan de acción conveniente con respecto a los medios y fines institucionales [3].

El enfoque sistémico es una visión holística, despliega variedad interpretativa, comprende el enfoque crítico, el interpretivismo y la actitud práctica. El Enfoque Sistémico permite el acceso a lo complejo y lo permite sin desbaratar su integridad como tal. Entonces, ve la cosa en el juego de sus interrelaciones [4].

Dicho enfoque no solo diagnostica a un grupo social, autoridades públicas y entidades de apoyo, sino que también puedo delinear respuestas que apuntaron a la aclaración de preguntas oscuras que generan asistencia financiera y otros para la formulación de acciones eficientes para el desarrollo de este sistema [5]. El enfoque sistémico para el diseño y el cambio organizacional es mediante: visión, misión, cultura, capacidad, aprendizaje; describe las diferentes aplicaciones de la comunidad para ilustrar su relevancia teórica y práctica para la investigación operativa comunitaria [6]. Según [7], describen una primera fase como el "descubrimiento", de una intervención de investigación-acción participativa sistémica "en los sistemas de gestión de una organización" de desarrollo de comunidad rural en la provincia de KwaZulu-Natal de Sudáfrica; los objetivos de la intervención fueron: mejorar el sistema de gestión de esta organización, y evaluar la utilidad de metodologías de sistemas particulares para el mejoramiento de los sistemas de gestión de organizaciones involucradas en el desarrollo comunitario en contextos rurales con pocos recursos.

La Cibernética Organizacional [8], una metodología con enfoque sistémico [9], según [10], describe "las condiciones necesarias y suficientes para que una organización sea viable, cuando éstas no se cumplen, las consecuencias, según la gravedad de la carencia, son un inadecuado funcionamiento o incluso la desaparición de la organización". La cibernética organizacional utiliza el modelo de sistema viable de sigla MSV, modelo que sintetiza las funciones o sistemas de una organización viable [11], [12], estos son: operación, coordinación, cohesión, auditoría, adaptación e identidad-política. Los casos documentados cubren una amplia gama de aplicaciones, subrayando así el gran potencial del MSV; los estudios de caso ejemplifican la fuerza sin paralelo del modelo como un marco conceptual para un mejor diagnóstico y diseño organizativo $\mathrm{y}$, por lo tanto, pueden utilizarse con fines didácticos [13]. El MSV, utilizado en la cibernética organizacional en diagnóstico y diseño organizacional, intenta brindar las condiciones necesarias y suficientes de viabilidad para cualquier sistema que reconozca su propia identidad y, al hacerlo, invita ese reconocimiento por parte de otros [8].

La cibernética organizacional es capaz de comprender las complejidades externas e internas de las organizaciones, este enfoque es un esquema para diagnosticar y diseñar la estructura organizativa [14]. Es considera que el sistema de control de gestión proporciona un control eficaz, lo que significa que equilibra adecuadamente la variedad del sistema y el entorno, lo que permite a las sucursales alcanzar sus objetivos de rendimiento de manera consistente [15].

El diagnóstico organizacional mediante el enfoque sistémico-cibernético propone en términos de [16], el proceso de ayuda a la alta dirección de la universidad a diagnosticar la situación al comienzo de su mandato y a diseñar acciones pertinentes; el proceso comienza aclarando la identidad, el propósito y los límites de la universidad, y luego identifica los niveles estructurales requeridos. Para cada uno de ellos se exponen los factores clave a considerar y las acciones a tomar. El uso de una Matriz de Factores Claves de Niveles de Recursión ayudó a mantener una visión coherente y holística de la intervención. En el caso de [17], los hallazgos evidencian algunos ejemplos de problemas operativos que fueron expuestos a través del proceso de intervención y muestra algunas conclusiones generales para respaldar el uso del MSV para analizar operaciones y respaldar la viabilidad de pequeñas empresas. Una serie de temas de "amenazas a la viabilidad" fueron evidentes y discutidas. Del mismo modo, existe un creciente interés en modelos organizativos alternativos entre los directores ejecutivos, ya que resulta obvio que los modelos clásicos existentes ya no podían hacer frente a la complejidad y la dinámica de los mercados del siglo XXI; el modelo de sistema viable les proporciona un modelo para distinguir lo correcto de lo incorrecto en las preguntas organizacionales; también, proporciona un idioma que debe aprenderse en las condiciones típicas y desafiantes de aprender cualquier idioma nuevo. La mayoría de los gerentes superan este obstáculo rápidamente y encuentran un modelo confiable no demasiado difícil de aplicar para velocidad, dirección y precisión en el diagnóstico y diseño de sus organizaciones [18]. En adición, [19] proponen, a partir de la forma en que un médico alopático hace un diagnóstico de la salud de una persona, una metáfora del diagnóstico organizacional que puede contribuir en la búsqueda de una forma cada vez más integral de calificar a una organización como saludable. En forma similar, existe una variedad de condiciones y factores patológicos que afectan el desempeño organizacional (del sistema), sin embargo, faltan métodos de apoyo que puedan guiar a los profesionales en la identificación de patologías que existen en los sistemas bajo su competencia [20].

Las patologías y arquetipos cibernéticos sistémicos organizacionales coadyuvan el diagnóstico organizacional. Las patologías estructurales están relacionadas con un abordaje inadecuado de la complejidad total enfrentada por cualquier organización [21]. En "función del tamaño de la organización y de su entorno relevante, el entorno podría dividirse en sub-entornos y al mismo tiempo, desagregar la organización en sub-organizaciones que limiten su 
actividad a los sub-entornos" [10]. Mientras, las patologías funcionales, evidencian si las cinco funciones sistémicas necesarias para la viabilidad de la organización están favorablemente representados y funcionan en forma apropiada [21]. De la misma forma, los arquetipos de identidad revelan organizaciones que tienen transformaciones poco claras, las "discrepancias entre el discurso utilizado y el comportamiento observado dificulta reconocer su identidad" [22]. Así también, los arquetipos estructurales revelan una utilización inadecuada de recursos y una pobre regulación de las actividades organizacionales [22]. Al explorar el potencial del uso combinado del MSV y el análisis de redes sociales para identificar patologías organizacionales, proporciona evidencia empírica de los beneficios del uso combinado de análisis de redes sociales para mejorar la identificación de patologías organizacionales en las intervenciones de la cibernética organizacional, al proporcionar un marco cualitativo y cuantitativo adicional para la interpretación de los hallazgos provenientes del diagnóstico organizacional utilizando el modelo de sistema viable [23].

El propósito del trabajo es diagnosticar el sistema organizativo en una institución de educación superior universitaria mediante el enfoque sistémico-cibernético y procesos apoyado en patologías y arquetipos cibernéticos organizacionales. Ello permitirá implementar sistemas de gestión bajo las exigencias de la Ley del SINEACE, y norma ISO 9001:2015.

\section{Materiales y Métodos}

La investigación fue realizada en una institución de educación superior universitaria pública en Huancayo. Facultad de Ingeniería Metalúrgica y de Materiales (FIMM), Provincia de Huancayo, Junín, Perú.

El desarrollo de la metodología de investigación es mediante el diagnóstico sistémico - cibernético [24].

El diagnóstico sistémico-cibernético, al inicio, sintetiza el sentido y propósito de la organización en una definición sistémica; en seguida, desarrolla el desdoblamiento de complejidad, el diagnóstico de funciones sistémicas, el modelo del sistema viable a modo de diagnóstico y por último, evidencia las patologías y arquetipos organizacionales; mediante las siguientes actividades [25], [26], [27]:

- Declarar la definición sistémica.

- Desdoblar la complejidad organizacional.

- Diagnosticar las funciones sistémicas: autonomía S1, sincronización y estabilización S2, cohesión S3, auditoria y monitoreo $\mathrm{S} 3 *$, adaptación e inteligencia S4, identidad y ethos S5.

- Desarrollar el MSV modo de diagnóstico.

- Evidenciar las patologías y los arquetipos cibernéticos organizacionales.

- En forma complementaria, diagnosticar mediante las matrices de los estándares de acreditación del
SINEACE, las clausulas y requisitos de la NTP ISO 9001:2015 y el integro en la organización los criterios de viabilidad.

El Decano autorizó la intervención sistémica en la FIMM. La metodología de trabajo siguió consideraciones éticas nacionales e internacionales, la cual fue aprobado por el Concejo de Facultad.

\section{Resultados}

Al inicio, el diagnóstico de las funciones sistémicas, seguido de la identificación y análisis de las patologías y arquetipos organizacionales, al final, el diagnóstico del sistema de gestión de la calidad según la NTP ISO 9001:2015, el diagnóstico de acreditación modelo SINEACE, el diagnóstico general mediante el enfoque de sistema viable.

\subsection{Diagnóstico de las funciones sistémicas}

a) Diagnóstico de autonomía

La FIMM, está constituida por tres procesos misionales: Formación Profesional, Investigación y Extensión Cultural y Proyección Social, en respuesta a los requerimientos de la sociedad. Los procesos en la actualidad no cumplen su razón de ser de la Facultad, se desarrollan individualmente y presentan una serie de ataxias que generan el funcionamiento inadecuado de la Facultad.

El proceso Formación Profesional constituye uno de los procesos misionales de la FIMM, su principal propósito es "formar profesionales humanistas, competitivos, investigadores, líderes con principios y valores comprometidos en el desarrollo sostenible, mediante la transmisión de conocimientos en las clases", estas son desarrolladas de acuerdo al plan curricular, el cual es elaborado en base al perfil de ingresante, perfil de egresado, competencias, asignaturas y estrategias de evaluación; cubriendo así la demanda de formación profesional de los postulantes a la FIMM.

El proceso de Formación Profesional provee de capital humano, los estudiantes, a los otros procesos misionales Investigación, Extensión Cultural y Proyección Social. No obstante, es apreciado como un proceso con gran capacidad de autonomía, ya que no depende de los otros procesos para su autorregulación, toma sus decisiones en coordinación con los interesados (Docentes, Decano, Director de Departamento Académico).

El Departamento Académico es considerado la gestión local del proceso Formación Profesional, ya que es el ente que coordina con los docentes acerca de los horarios, la carga lectiva y otros asuntos. Es regido por el Reglamento Académico de la UNCP y el calendario de actividades académicas.

El Director del Departamento Académico revisa y evalúa los sílabos de todas las asignaturas, controla la asistencia de los docentes mediante un parte, de igual manera realiza visitas a las aulas de clases esporádicamente, 
para verificar la asistencia de los docentes y el avance silábico e informar al decano la situación de los docentes. Por otro lado se evidencia que los docentes no utilizan la Carpeta Académica el cual contiene documentos de avance de sílabos, asistencia de los alumnos y otros documentos; tampoco, manejan indicadores de rendimiento.

En el proceso de investigación, la Facultad cuenta con un área especializada en Investigación, tanto para docentes (Investigación Docente) y para estudiantes (Investigación Formativa), los trabajos de investigación en su mayoría, más del $90 \%$, son presentados por docentes. Su principal objetivo es registrar y evaluar trabajos de Investigación Docente e Investigación Formativa, dichos trabajos son realizados en respuesta a las necesidades de ciencia, tecnología e innovación de la sociedad y acorde a las líneas de investigación de la facultad. Así mismo es considerado un proceso autónomo ya que no depende de otros procesos dentro de la FIMM y es regido por el reglamento interno y calendario de investigación de la UNCP.

La relación con los otros procesos misionales es ambigua y no se evidencia el flujo de información entre estos. Formación Profesional provee de capital humano a Investigación, aun así, la cantidad de proyectos de investigación presentados por estudiantes es mínima.

Los estudiantes y docentes presentan los trabajos en primera instancia al Instituto Especializado de Investigación de la FIMM, como representante de la gestión local del proceso, quien a su vez debe comunicar y coordinar a nivel local (docentes o estudiantes realizando trabajos de investigación) y a nivel central (gestión central de la FIMM), sin embargo, en la actualidad la coordinación a nivel local es de forma verbal y la coordinación con la gestión central es solo de tramite documentario. Los docentes presentan sus informes de avances de acuerdo al cronograma de la UNCP, considerado como único documento oficial de coordinación y estabilización; no existen políticas ni normas dentro de la Facultad que guíen el proceso de Investigación.

No se realizan auditorias ni monitoreo internamente, por ende no existe evidencias documentales de la situación real de los trabajos de investigación que detallen el avance de las investigaciones, cumplimiento de plazos, logro de objetivos dentro de la FIMM. De igual manera, no cuenta con indicadores que midan el desempeño.

Los docentes realizan investigaciones de acuerdo a necesidades estudiadas previamente por ellos mismos, en reacción a situaciones problemáticas pasadas o de la actualidad, sin tener en cuenta el entorno y el futuro, ya que la FIMM no cuenta con documentos formales basados en estudios de los posibles problemas en el futuro. Así mismo en la mayoría de casos la presentación de trabajos de investigación y sus avances, es solo de cumplimiento normativo.

El proceso misional de Extensión Cultural y Proyección Social con sigla ECPS de la FIMM, tiene como propósito: "Difundir, registrar, evaluar los trabajos de
Extensión Cultural, Proyección Social y Transferencia Tecnológica", este proceso misional tiene las siguientes actividades: Desarrollar trabajos de ECPS, Evaluar los trabajos de ECPS. Las necesidades culturales y sociales son consideradas por la Facultad como el entorno directo para éste proceso misional.

La gestión de ECPS es responsable de difundir, registrar, evaluar y dar seguimiento a los trabajos de Extensión Cultural, Proyección Social y Transferencia Tecnológica.

La manera de rendir cuentas entre el entorno y operación se constituye por información proporcionada por el entorno que facilite la realización y cumplimiento de trabajos de ECPS, en la Facultad no existen otros que permitan a su operación obtener información de su entorno. Así también, la comunicación y rendición de cuentas entre su operación y la gestión se constituye por los informes de avance y final de los trabajo de Extensión Cultural y Proyección Social. Además, la operación comunica al entorno todo lo relacionado con el desarrollo de los trabajos de Extensión Cultural y Proyección Social.

La comunicación de la toma de decisiones por parte de la Gestión, es mediante lineamientos y directivas de ECPS provistos por la universidad.

La coordinación central es entre docentes y estudiantes, asimismo la Gestión de ECPS mantiene comunicación con el Decano a través de informes de gestión. Por otro lado, no hay evidencia de ningún tipo de monitoreo o auditoria interna. Incluso existe indiferencia por realiza estudios de impacto de las necesidades culturales y sociales en el ámbito de la Facultad. Por otro lado, la Gestión local de ECPS no mantiene comunicación con el Sistema 5 (Identidad y política) de la Facultad.

b) Diagnóstico de coordinación

La FIMM considera como mecanismos de coordinación lo siguiente: los horarios y el calendario académico para Formación Profesional, el cronograma de actividades de ECPS y el cronograma de actividades que señala los plazos de presentación de trabajos de investigación y sus avances para investigación. Sin embargo, es evidente que los procesos misionales no interrelacionan, ni coordinan entre ellos. Figura 1

c) Diagnóstico de monitoreo y seguimiento

La Facultad no evidencia la realización del monitoreo o auditorías internas a los procesos: Formación Profesional, Investigación, ECPS. Al proceso de Formación Profesional el Director de Departamento Académico visita esporádicamente los salones de clases para verificar la asistencia de los docentes y el avance silábico, sin embargo no hay documentos que consoliden dicha información. Por otro lado, solo existe control interno por parte de la Universidad mediante la Oficina de Control Interno. Figura 1 
d) Diagnóstico de cohesión

La función de control de la Facultad está conformada por el Decano, el Consejo de Facultad, y las comisiones permanentes y especiales, los procesos del Sistema 1 lo ven como autocrático, y se evidencia que Investigación y ECPS tienen mayor grado de libertad que Formación Profesional ya que se da el caso de que el Sistema 3 se involucre en el desarrollo de este proceso.

Por otro lado, el Sistema 3 traduce las políticas globales en planes operacionales a través de documentos de gestión administrativa (Proveído, memorándum, comunicados, etc.) o Resoluciones de Consejo.

La negociación de recursos con los proceso del Sistema 1 se da de la siguiente manera, las comisiones correspondientes realizan la gestión de provisión con el decano en la Oficina General de Planificación de la Universidad, existen demoras en la entrega de provisiones $\mathrm{y}$ muchas veces dichas gestiones quedan en stand by.

La revisión del rendimiento de los procesos del Sistema 1 lo realizan: El Decano, el Concejo de Facultad, la Oficina General de Administración Académica, la Oficina General de ECPS y la Oficina General de Investigación de la Universidad Nacional del Centro del Perú. Figura 1

e) Diagnóstico de inteligencia y adaptación

En la Facultad, la función de inteligencia es realizada por la Comisión de Planificación y Presupuesto, existe indiferencia a las novedades y tendencias, ya que cumplen las funciones según lo soliciten las oficinas de la Universidad. Realizan Planes Operativos anualmente con poca evidencia de resultados favorables, solo lo realizan por obligación legal.

Por otra parte, no hay evidencia de monitoreo del entorno de la Facultad ni mucho menos de la evolución de las tendencias. La planificación es indiferente a la novedad y solo está enterado de los últimos acontecimientos mediante canales formales los cuales son informados por el Decanato. Asimismo, evidencia que la toma de decisiones está centralizada en el Decano y el Consejo de Facultad.

De la misma forma, existe indiferencia en el procesamiento, filtración y distribución de la información relevante pertinente a la Facultad. Figura 1

\section{f) Diagnóstico de identidad y política}

Los responsables de las políticas de la Facultad son el Decano y la Junta de Facultad, los procesos que pertenecen a dicho sistema (sistema 5) es el Decanato, no existe flujo de información acerca del entorno y el futuro, ni de la situación real de los procesos misionales. La identidad suministrada es inapropiada y no expresan propósitos claros de la FIMM, es evidente también que no comparten la misma visión con docentes y estudiantes esto impide el desarrollo u operación de los procesos encargados de hacer estudios del entorno y el futuro. De igual manera, existe indiferencia de estabilizar y enfatizarse en los cambios, los responsables demuestran que estar desorganizados para actuar creativamente, casi la totalidad de los procesos operan con el fin de cumplir la normatividad de la UNCP, este sistema funciona como un tramitador de documentos. Figura 1

La Tabla 1, detalla la función sistémica y el componente o proceso correspondiente en la Facultad. Asimismo, es notorio la ausencia o inexistencia del Sistema 2 y Sistema $3 *$.

Tabla 1.

Análisis de Funciones Sistémicas - Regulación

\begin{tabular}{|c|c|}
\hline SISTEMA & FIMM \\
\hline Sistema 1: Operación & $\begin{array}{l}\text { Formación Profesional } \\
\text { Investigación } \\
\text { Extensión y Proyección Social }\end{array}$ \\
\hline $\begin{array}{l}\text { Sistema 2: Coordinación y } \\
\text { estabilización }\end{array}$ & -- \\
\hline Sistema 3: Cohesión & $\begin{array}{l}\text { Decano } \\
\text { Consejo de Facultad }\end{array}$ \\
\hline $\begin{array}{l}\text { Sistema 3*: Auditoria y } \\
\text { Monitoreo }\end{array}$ & -- \\
\hline $\begin{array}{l}\text { Sistema 4: Adaptación e } \\
\text { Inteligencia }\end{array}$ & $\begin{array}{l}\text { Comisión de Planificación, } \\
\text { Economía y Evaluación }\end{array}$ \\
\hline Sistema 5: Identidad y Política & $\begin{array}{l}\text { Decano } \\
\text { Junta de Facultad }\end{array}$ \\
\hline
\end{tabular}

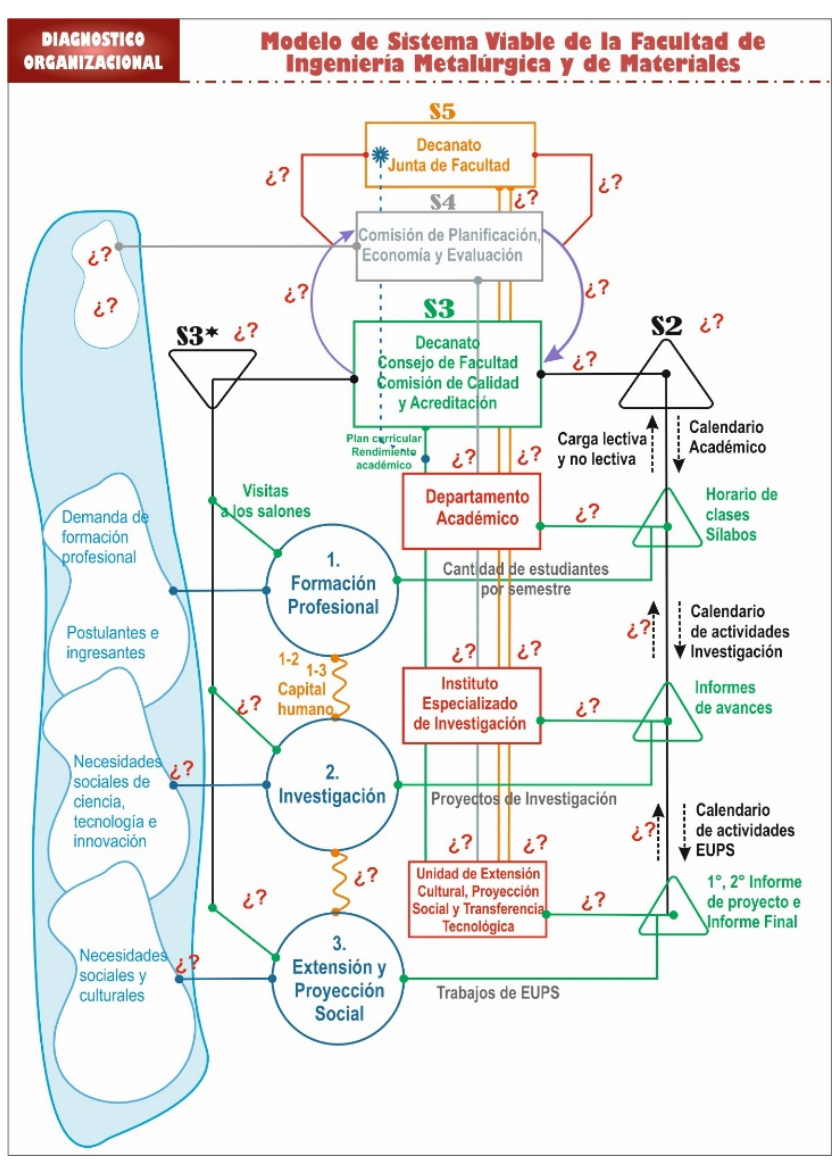

Fig. 1. Modelo de Sistema Viable de la FIMM (diagnóstico) 


\subsection{Patologías y Arquetipos Organizacionales}

a) Patologías organizacionales

Ausencia de niveles de recursión en niveles intermedios, desconocimiento del entorno al que están atendiendo los procesos de Investigación y ECPS, solo es evidente el entorno de Formación Profesional. Figura 2

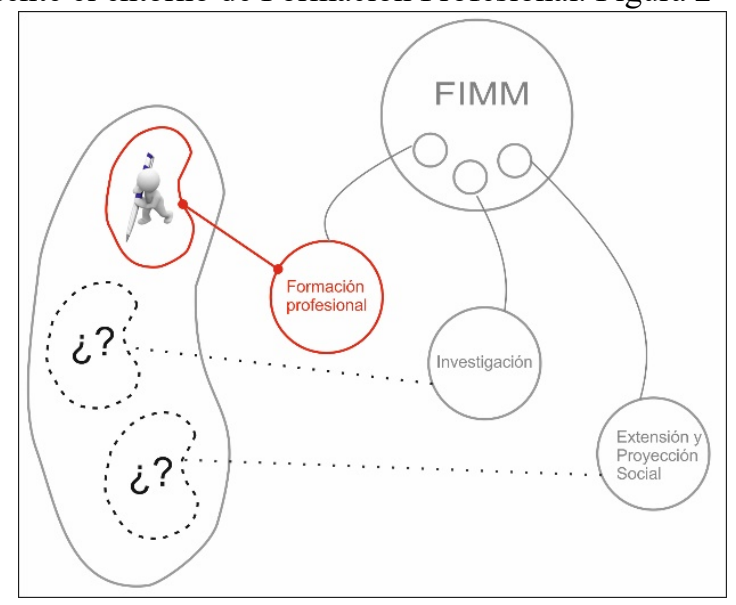

Fig. 2. Patología ausencia de nivel de recursión

Desdoblamiento confuso, patología estructural, en la Facultad, los procesos Investigación y ECPS rinden cuentas a la Oficina General de Investigación de la UNCP y la Oficina General de ECPS de la UNCP respectivamente, más no a la FIMM quien es un intermedio. Por lo cual se evidencia la falta de comunicación de ida y vuelta entre el nivel 1 y nivel 2. Figura 3

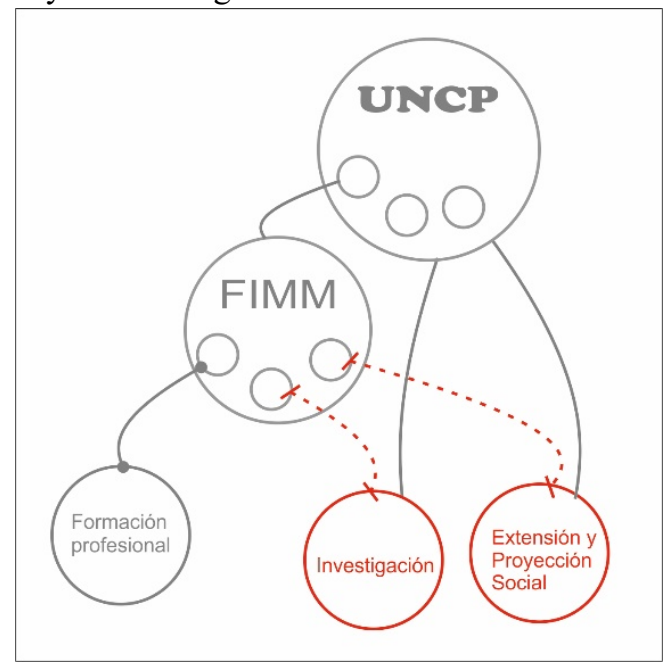

Fig. 3. Desdoblamiento confuso

Identidad mal definida, patología funcional, la misión de la Facultad es distinto a la identidad encontrada en los procesos principales y como operan estas. No es coherente entre la "misión" y lo que hace (Sistema 1). Figura 4

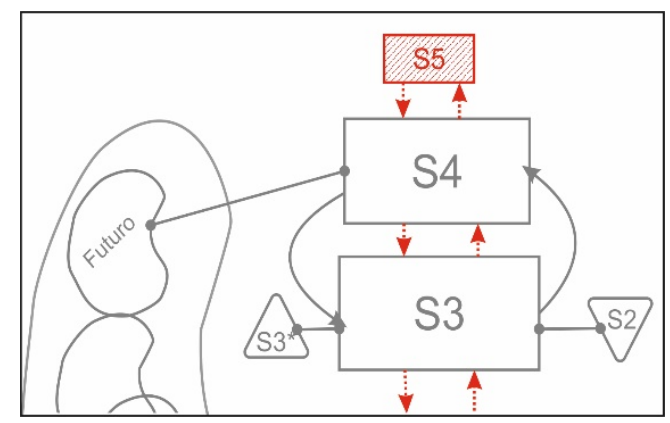

Fig. 4. Identidad mal definida

Esquizofrenia institucional, patología funcional, existen diferentes puntos de vista de la Facultad en cuanto a lo Laboral, Enseñanza- Aprendizaje y Normativa. Figura 5

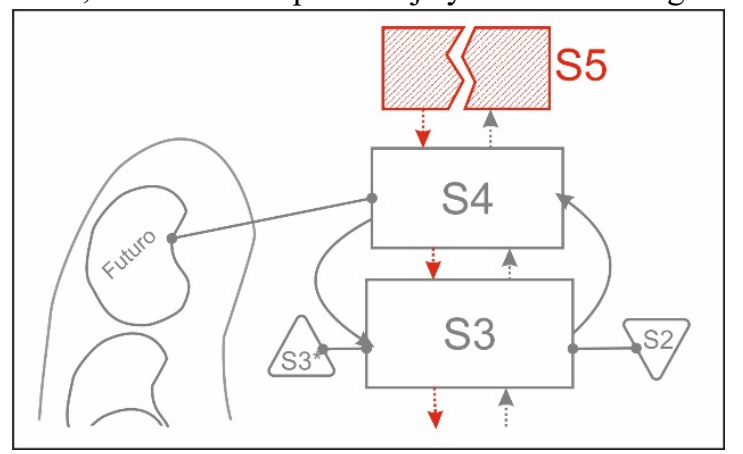

Fig. 5. Esquizofrenia institucional

Colapso del S5 en el S3, patología funcional, las decisiones son tomadas por la autoridad que tienen el Decano y Consejo de Facultad, muchas veces se olvidan de optimizar el día a día. Es decir la gestión de Formación Profesional, Investigación y ECPS. Figura 6

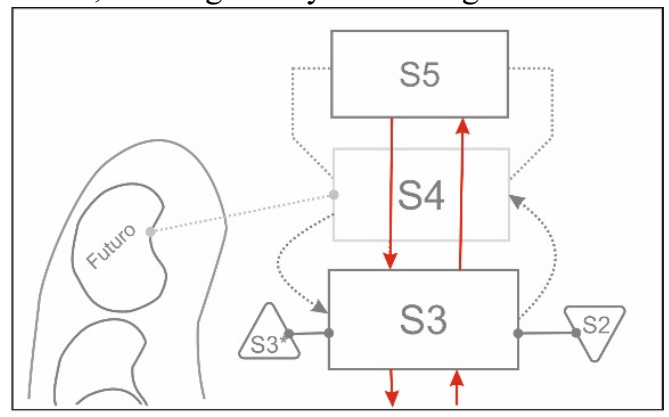

Fig. 6. Colapso de S5 en S3

Ave sin cabeza, patología funcional, las labores de la Comisión de Planificación, Economía y Evaluación centra atención en cumplir lo exigido legalmente dejando de lado la prospección del futuro, los estudios de su entorno y la transmisión de los planes de mejora. La disponibilidad de información es nula, hay ausencia del análisis histórico y la inexistencia de información de que va pasar si la Facultad sigue por el mismo camino. Figura 7 


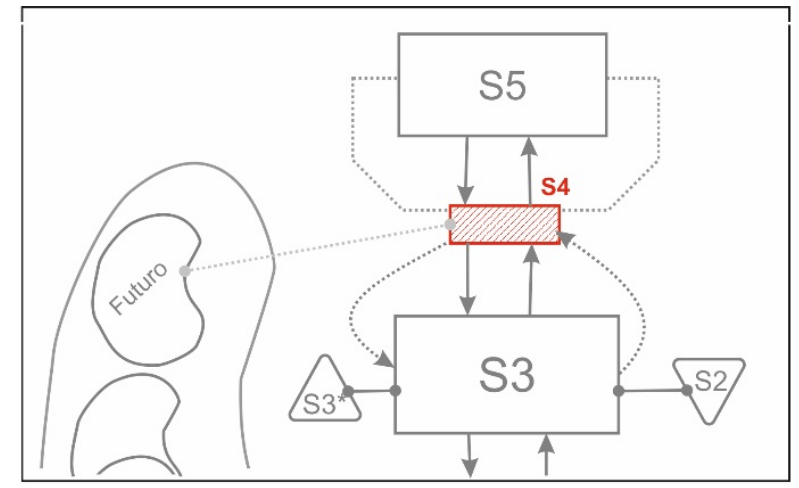

Fig. 7. Patología ave sin cabeza

Disociación entre el Sistema 4 y Sistema 3, patología funcional, inexistente consideración en utilizar la información estratégica e indiferencia en transmitir tendencias a ser incorporadas en la Facultad. Los canales de información son deficientes entre el S4 y las operaciones: Formación Profesional, Investigación y ECPS; el tiempo de acceso a la información es disconforme y no permite tomar decisiones en el momento pertinente. Figura 8

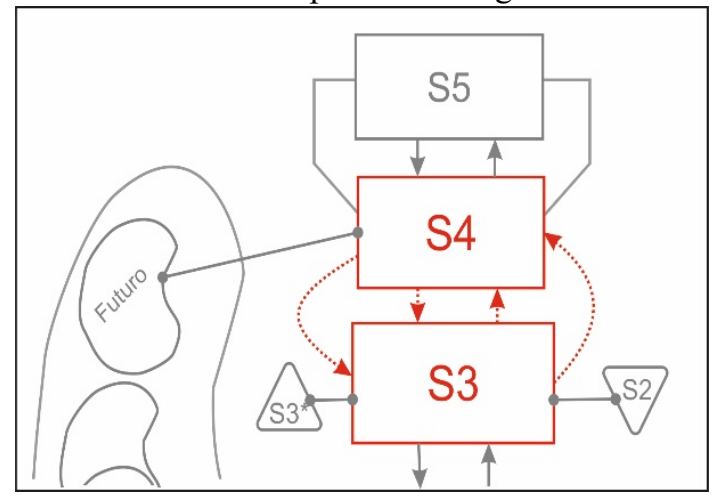

Fig. 8. Disociación entre S4 y S3

Estilo de dirección inadecuado, el Decano resguarda la Formación Profesional y poca cohesión para Investigación y ECPS. Figura 9

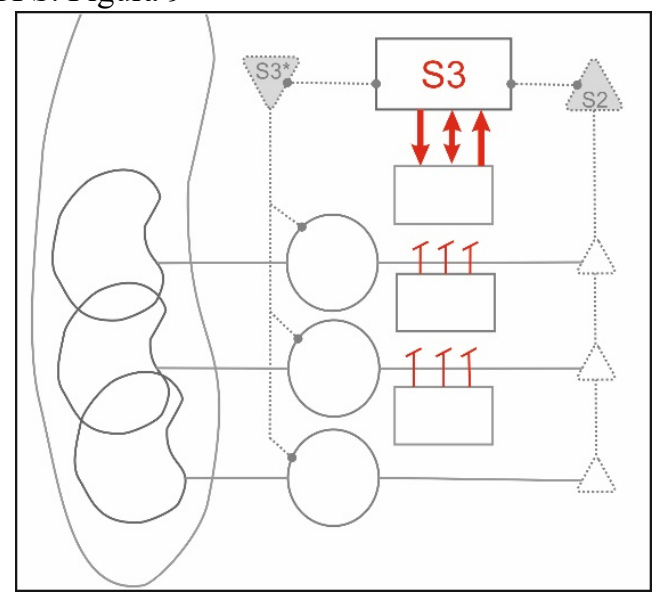

Fig. 9. Estilo de dirección inadecuado

Sistema 3 esquizofrénico, el Decano y el Consejo de Facultad descuidan canalizar la información del día a día de las unidades operativas y existe una interpretación de manera desatinada de las instrucciones del S5. Figura 10

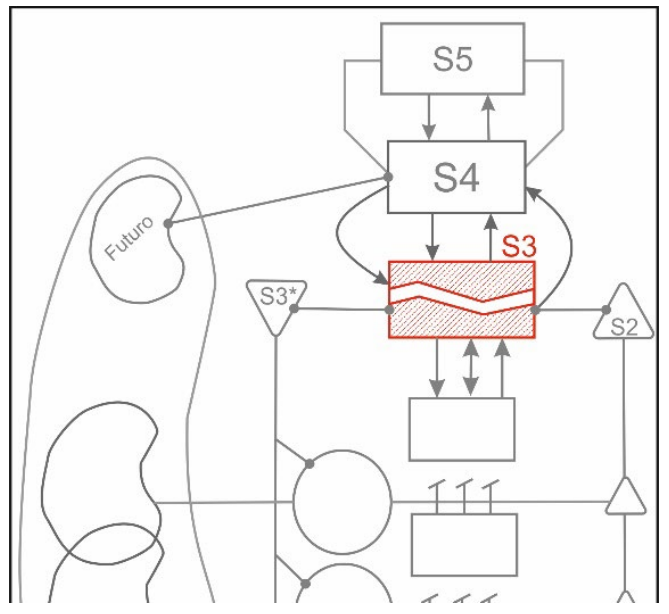

Fig. 10. Sistema 3, esquizofrénico

Conexión débil entre S3 y S1, la FIMM utiliza como únicos medios de comunicación, los documentos administrativos (oficios, memorándum, solicitudes y otros) $\mathrm{y}$ hace el intento de cohesionar e integrar al S1. Figura 11

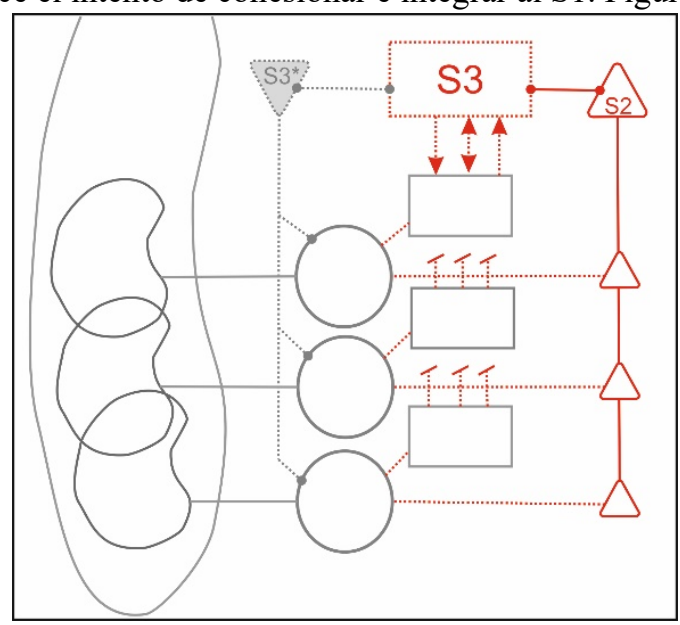

Fig. 11. Conexión débil entre S3 y S1

Hipertrofia de cohesión (S3), existe una dirección autoritaria, no tiene la variedad requerida para absorber la información del $\mathrm{S} 1$, originando la disminución de autonomía del S1 de la Facultad. Figura 12

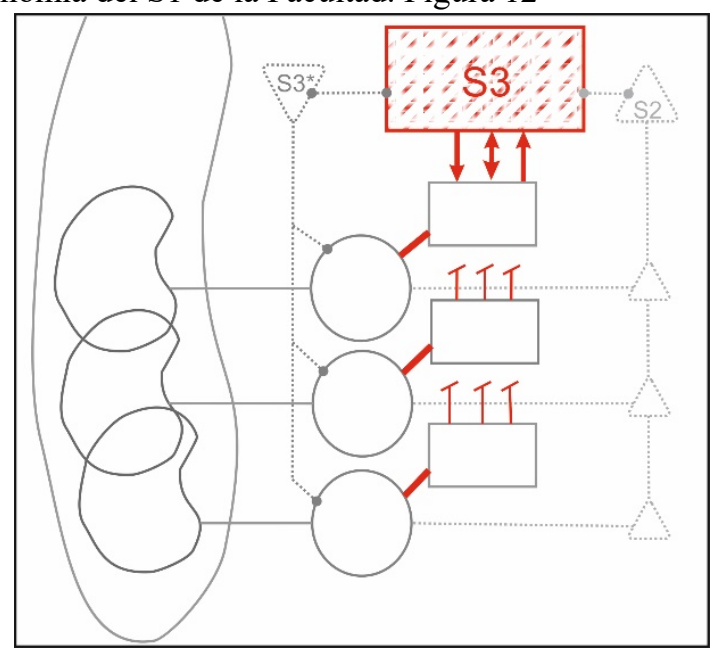

Fig. 12. Hipertrofia del Sistema 3 
Ausencia o desarrollo insuficiente del S3*, auditoriamonitoreo, patología funcional, indiferencia en realizar auditorías y monitoreo para verificar el estado de las unidades operativas (recursos, resultados, entre otros). Por resultante, corren el riesgo de que la información proporcionada por las unidades operativas sea falsa. Figura 13

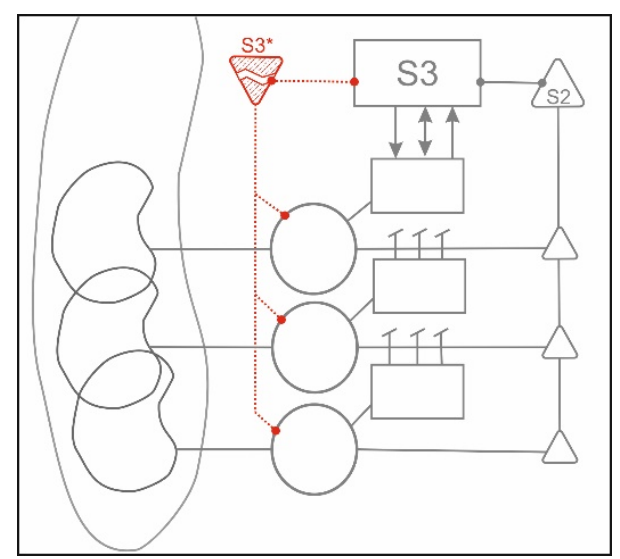

Fig. 13. Ausencia o desarrollo insuficiente del Sistema $3 *$

Comportamiento fragmentario al interior de la operación, Sistema 1 (Cada uno a los suyo), existen posibles coordinación locales en la Facultad, como el calendario de actividades de ECPS, de Investigación y de Formación Profesional. Por otro lado, no evidencia una relación directa, como las reuniones entre los responsables de las unidades operativas.

Bestias Autopoieticas, en los últimos tiempos la Facultad se ha preocupado por atender solo los requerimientos de Formación Profesional, quien posee mayor atención y dominio, por ende está más desarrollado en comparación de las otras unidades operativas. Formación Profesional está conformado por plan curricular, matriculas, emisión de certificados entre otros, a comparación con Investigación solo está conformado por Investigación Docente e Investigación Formativa, sin dejar de lado Extensión y Proyección Social que realizan solo sus proyectos en conjunto. Figura 14

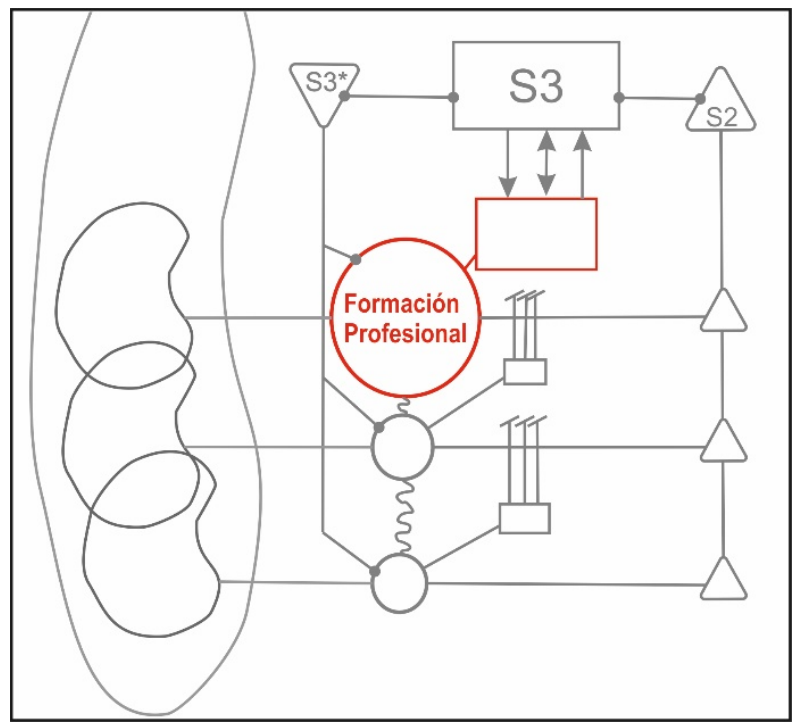

Fig. 14. Bestia autopoietica

Inexistencia del Metasistema, existe más atención en el Sistema 1 (E-A, I, EC, PS y TT) que en el desarrollo del metasistema (S3, S4 y S5). Cada proceso operativo misional trabaja según sus objetivos para conseguir sus propios resultados, dejando de lado el propósito de la FIMM en general. Figura 15

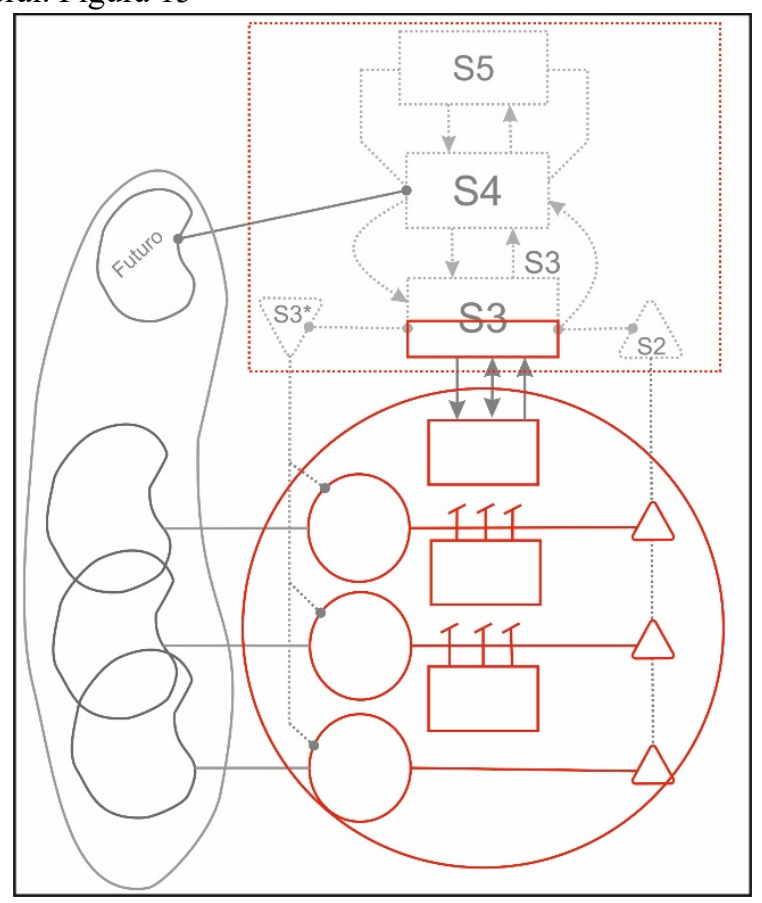

Fig. 15. Inexistencia del metasistema

Fragmentación de los Sistemas de Información, existe un software solo para el proceso formación profesional, "GALILEO", el cual sirve para ingreso y reporte de notas y asistencia y realizar pre matrículas de los estudiantes, el cual no interrelaciona con las otras partes de la Facultad. Figura 16 


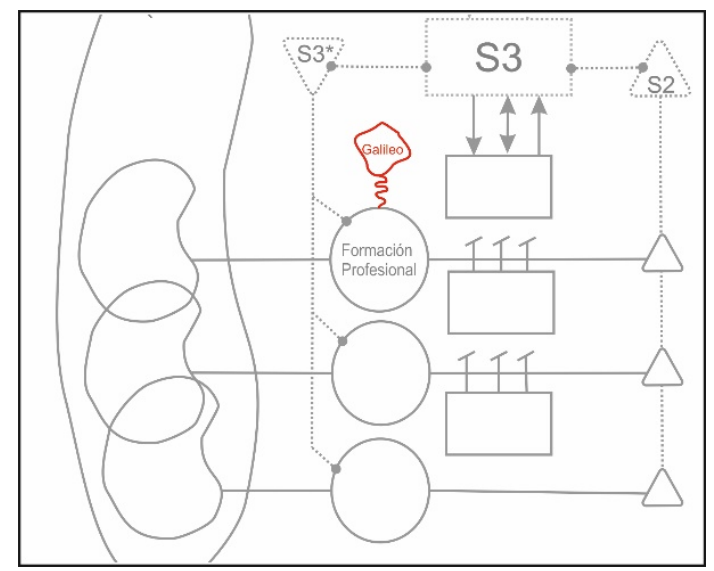

Fig. 16. Fragmentación de los sistemas de información

Ausencia de canales de información esenciales, la comunicación en su mayoría es mediante medios escritos, es decir, los documentos administrativos de gestión. Así también, utilizan los medios verbales. Figura 17

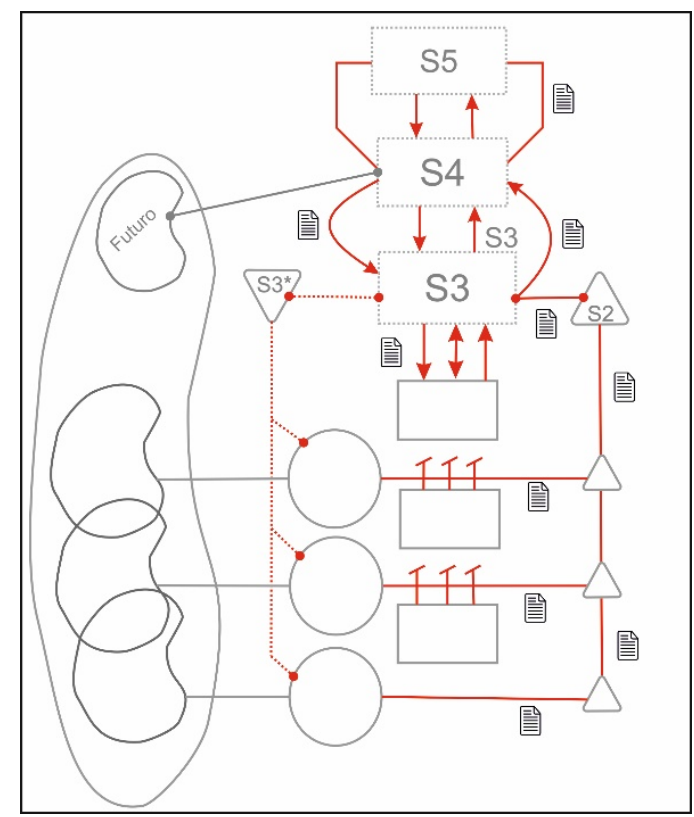

Fig. 17. Ausencia de canales de información esenciales

b) Arquetipos Cibernéticos Organizacionales

Arquetipo de Identidad, la identidad del Facultad está dominada por el proceso Formación Profesional y deja de lado la Investigación y ECPS. Figura 18

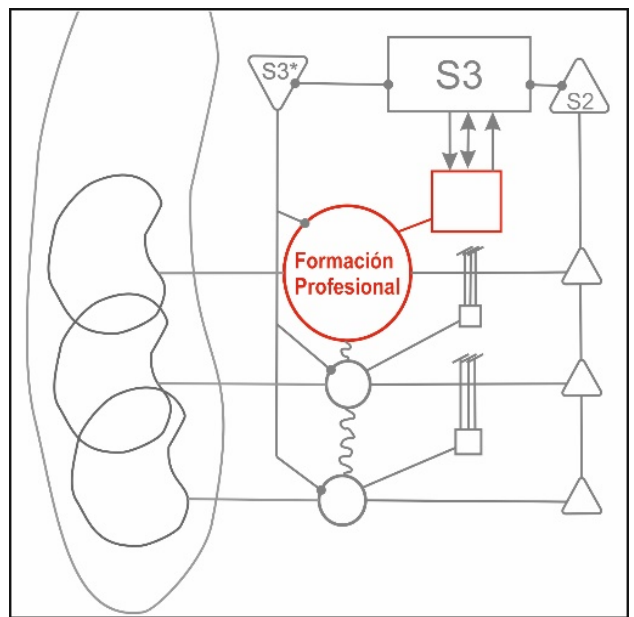

Fig.18. Arquetipo de identidad

Arquetipo de Propósito en Uso Diferente al Propósito Declarado, se considera que la identidad de la FIMM es desarrollar Investigación, ECPS y Formación Profesional, sin embargo, la FIMM no evidencia la preocupación por las dos primeras, solo se concentran en desarrollar formación profesional. Por otro lado, la FIMM brinda capacitación en ofimática, esto hace que pierda su identidad. Figura 19

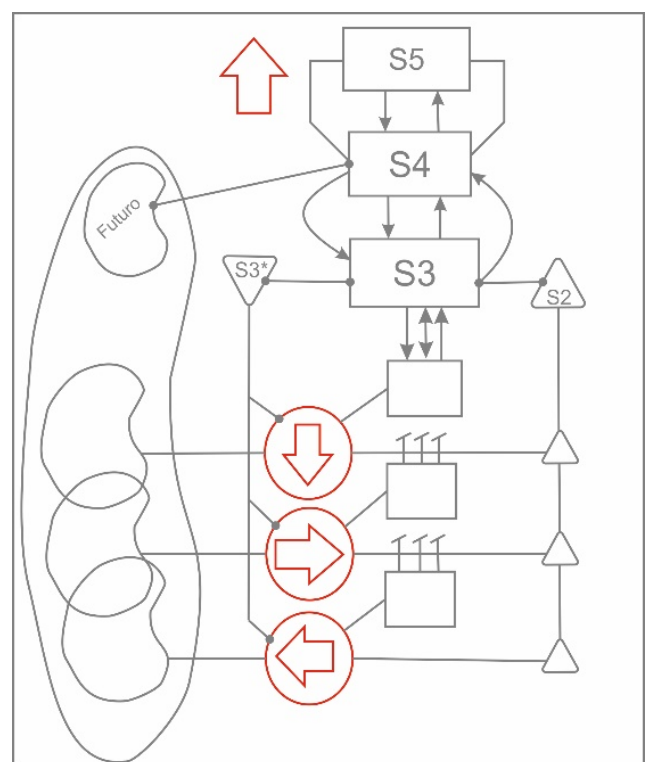

Fig. 19. Arquetipo de propósito en uso diferente al desarrollado

Arquetipo de Compatibilidad entre Formal con lo NoFormal, la estructura organizativa descrita en la ley, y el estatuto de la UNCP es diferente a la que viene realizando la Facultad, ello es evidente en los resultados de Investigación y ECPS.

Arquetipo de Sinergia Negativa, al juntarse las procesos misionales de la Facultad: Formación Profesional, Investigación y ECPS; no generan valor a la Facultad. Cada quien quiere lograr su propio propósito. Figura 20 


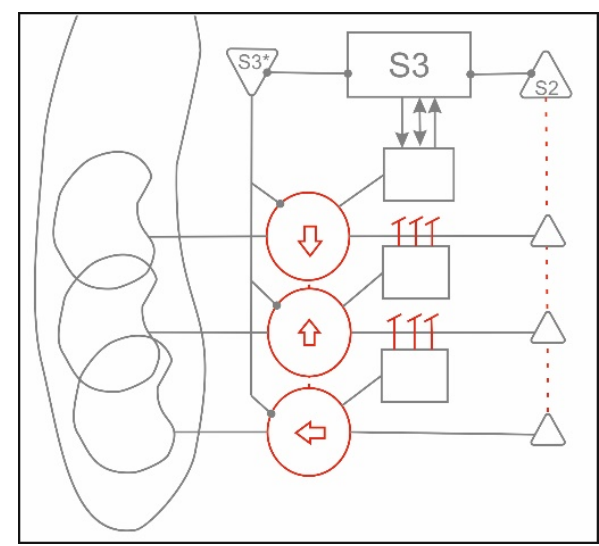

Fig. 20. Arquetipo sinergia negativa

Arquetipo de Identidad Fluida, la Facultad no puede esperar a que sus servicios permanecerán válidos por demasiado tiempo. La tecnología y también las expectativas de las personas cambian demasiado rápido; los estudiantes y grupos de interés cambian sus requisitos y necesidades con respecto a la Facultad. Figura 21

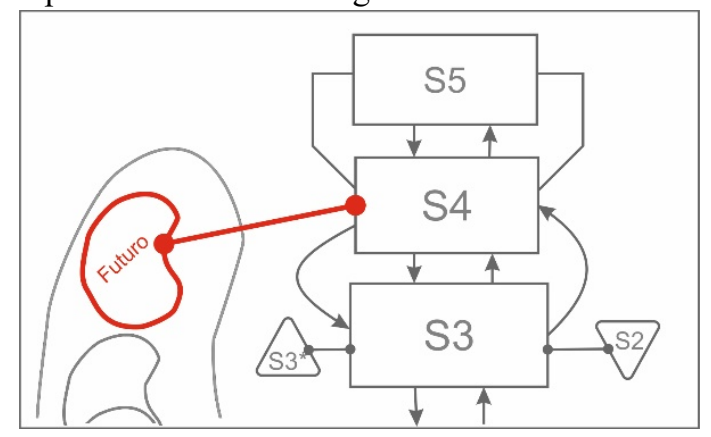

Fig. 21. Arquetipo de identidad fluida

Arquetipo Jerárquico, el Decano se involucra en las decisiones locales del S1: Formación Profesional, ECPS e investigación. Por otro lado, el Decano formula las políticas, pero el sistema 1 no los entiende. Figura 22

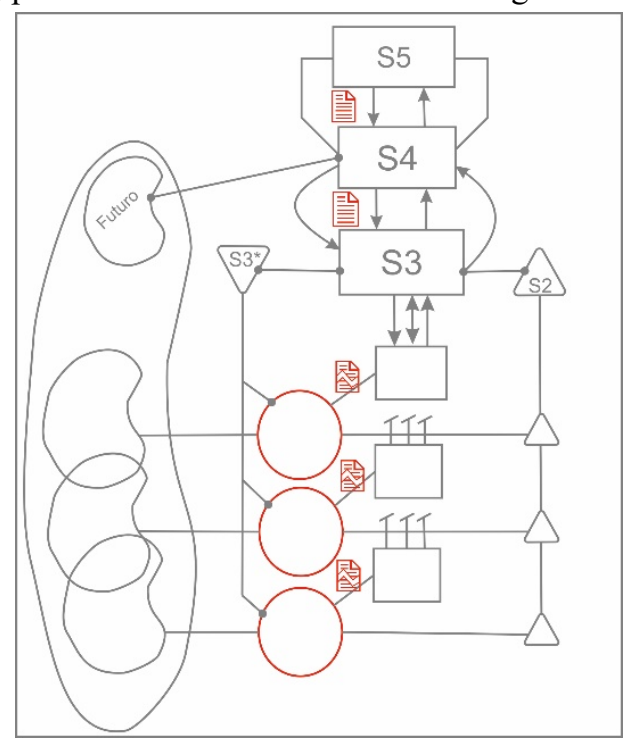

Fig. 22. Arquetipo jerárquico

Arquetipo de Competitividad Baja, en la Facultad cada uno de los requerimientos son atendidos independientemente a sus entornos por los procesos misionales. No quieren desarrollar su propia identidad, creen que viven al éxito y a expensas de Formación Profesional. Figura 23

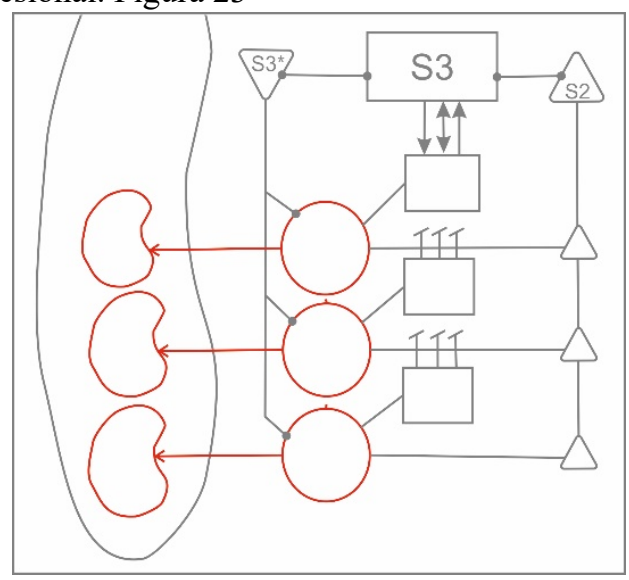

Fig. 23. Arquetipo de complejidad baja

Arquetipo de Politiquería, el Decano (sistema de gestión central de la FIMM) toma decisiones a nivel local (sistema 1), porque cree que no hay resultados favorables y no confía en la capacidad de las personas. Ejemplo: Investigación, ECPS, los reglamentos vienen de arriba y no son comunicados a todos, solo a los "interesados" (el Cronograma de Investigación solo al Director de Investigación), estos están elaborados y alineados a lo que dice la UNCP. Figura 24

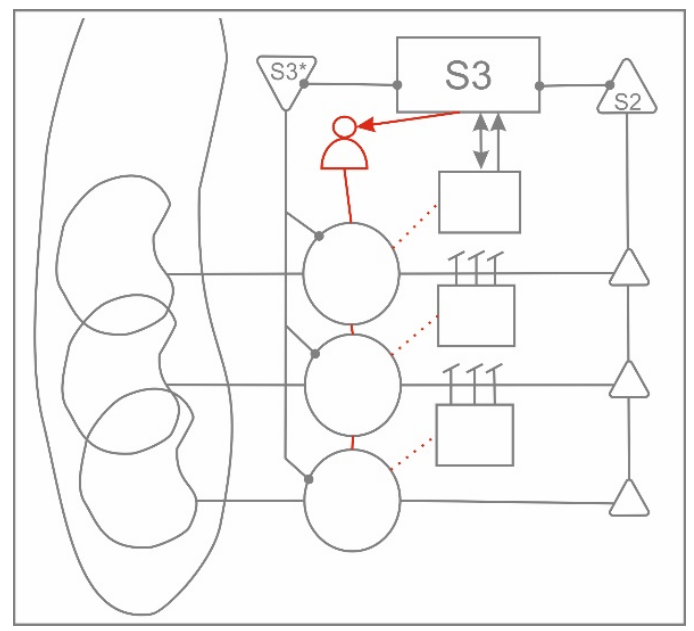

Fig. 24. Arquetipo de politiquería

Arquetipo de Auto Organización no-soportada, la gestión central de la Facultad desconoce cómo organizarse frente a las demandas del entorno, como la Acreditación y Gestión de la Calidad, alineamiento a la Ley Universitaria. Incapacidad para enfrentar el entorno con su estructura organizacional establecida. Figura 25 


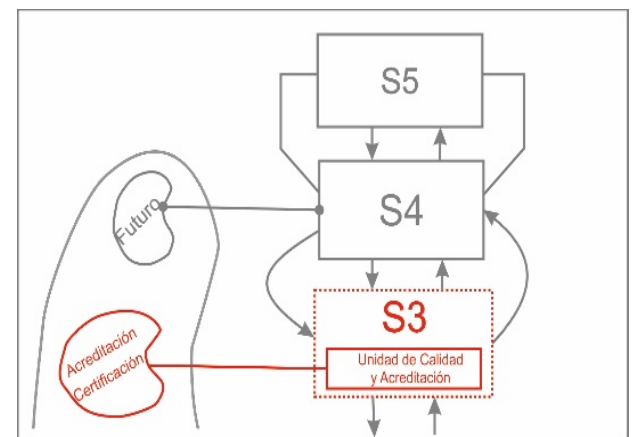

Fig. 25. Arquetipo de auto-organización no soportada

Arquetipo de Dilema de Control, inexistencia de canales de seguimiento y coordinación en la Facultad. No logra sus propósitos. Existe sobrecarga de información, incapacidad para procesar la información. En consecuencia los procesos misionales (Formación profesional, Investigación, ECPS) para salvar la situación presentan documentos con información poco verídicas. Figura 26

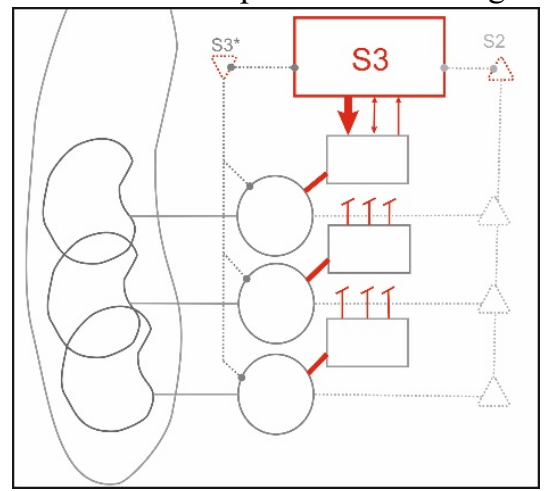

Fig. 26. Arquetipo dilema de control

Arquetipo de No Monitoreo, la información solicitada no es revisada por la Gestión Central de la FIMM, la información brindada es asumida como correcta, Ejemplo: en Investigación, los informes son enviados al Decano y después a la Oficina de la UNCP, en muchos casos el Decano no lo revisa y lo envía como está. Figura 27

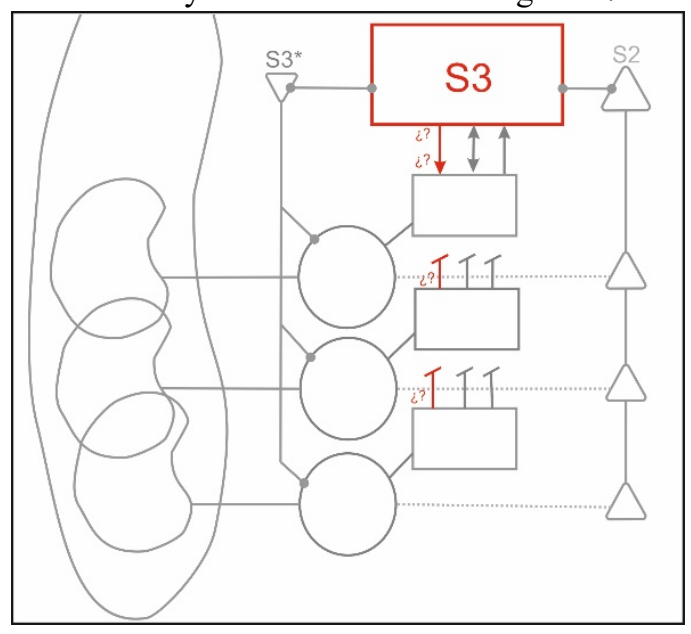

Fig.27. Arquetipo de no monitoreo

Arquetipo de Globalización, en la internacionalización de la Facultad, los requerimientos de sus grupos de interés y otras exigencias, está tratando de responder a los requerimientos del entorno que la Facultad dejando de lado su razón de ser como el servicio de Formación Profesional. Figura 28

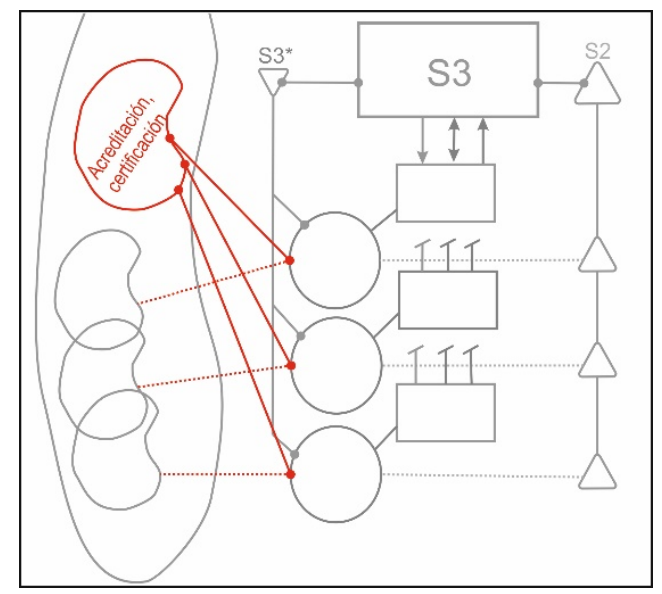

Fig. 28. Arquetipo de globalización

Arquetipo de Quiebre de Procesos Organizacionales, Las unidades orgánicas de la FIMM solicitan requerimientos para el funcionamiento de sus procesos, estos requerimientos deben tener el visto bueno del Decano, es evidente la demora en la respuesta de los documentos, lo cual dificulta en su proceso de presentación a la Oficina correspondiente en la UNCP. Figura 29

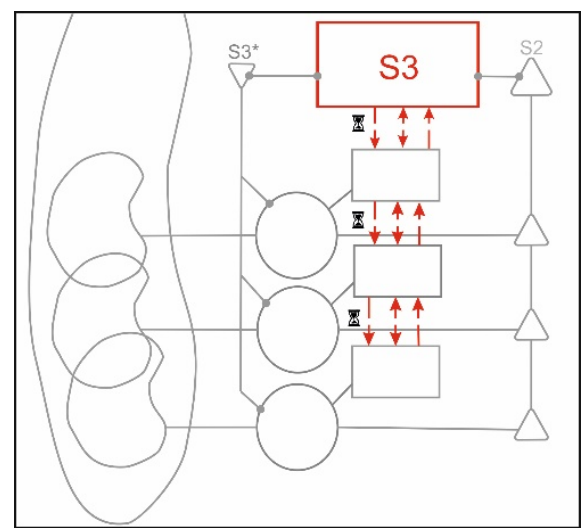

Fig. 29. Arquetipo quiebre de procesos organizacionales

Arquetipo de Centralización de Recursos, la provisión de los recursos es por parte de la UNCP, la respuesta para los requerimientos solicitados tienen mucha demora. Figura 30

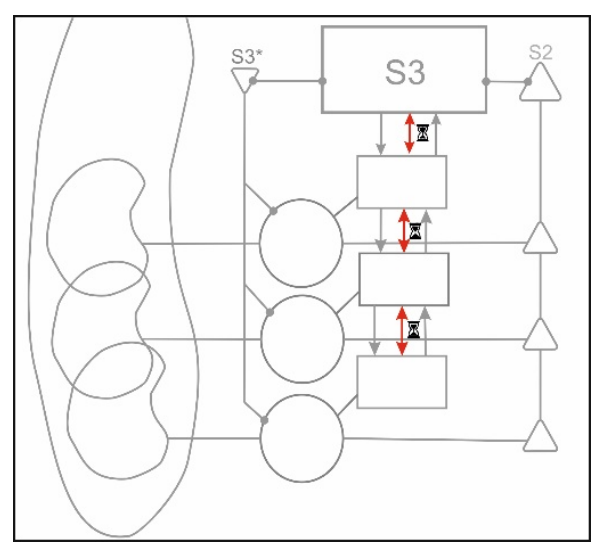

Fig.30. Arquetipo de centralización de recursos

Arquetipo de Actividad Primaria Dirigente, la mayor parte de los recursos de la Facultad son dirigidos para 
Formación Profesional. La mayoría de requerimientos son presentados por esta unidad operativa, mientras tanto Investigación, ECPS son tramitadores documentarios.

Arquetipo de Adaptación Débil, el entorno de la Facultad es muy exigente y fuerte, la FIMM desconoce sus grupos de interés, no realiza estudios acerca de su entorno y si los hay, solo responde a su entorno presente. Figura 31

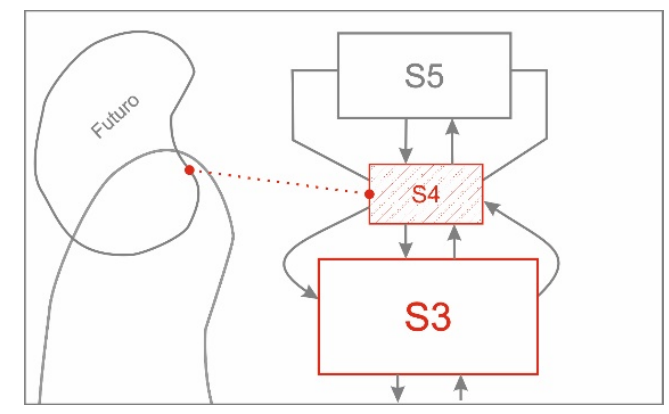

Fig. 31. Arquetipo de adaptación débil

Arquetipo de Pertenencia Organizacional, existen diferentes puntos de vista acerca del propósito de la FIMM estos son: la Facultad como centro laboral, desde el punto de vista legal y normativo, la Facultad como responsabilidad social y como institución pública universitaria. Figura 32

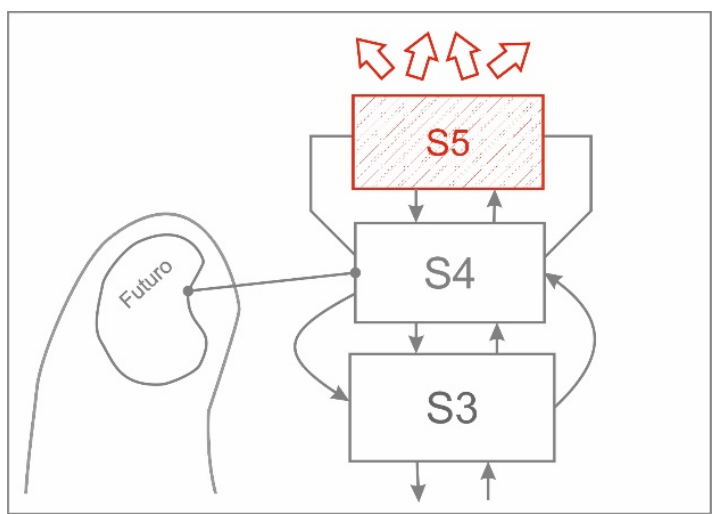

Fig. 32. Arquetipo de pertenencia organizacional

\subsection{Diagnóstico del sistema de gestión de calidad, Norma ISO 9001:2015}

El diagnóstico describe la situación actual de la FIMM, también, identifica oportunidades de mejora en relación al Sistema de Gestión de Calidad basado en NTP ISO 9001:2015.

Fue necesario realizar entrevistas a los integrantes de la FIMM, revisar documentos y normas internas, de acuerdo a un programa de entrevistas y recojo de información. Del mismo modo, definir dos maneras de presentar la información, una cualitativa, al mostrar evidencia escrita y análisis documental y otra cuantitativa, al evidenciar una calificación de porcentaje de avance e implementación. Tabla 2 y Tabla 3
Tabla 2

Valoración NTP ISO 9001:2015

\begin{tabular}{lrl}
\hline Criterios de Calificación & Puntaje & \multicolumn{1}{c}{ Estado } \\
\hline A $\quad \begin{array}{l}\text { Cumple } \\
\text { completamente con } \\
\text { el criterio enunciado }\end{array}$ & 10 & $\begin{array}{l}\text { Se establece, } \\
\text { se implementa y } \\
\text { se mantiene }\end{array}$ \\
\hline B $\quad \begin{array}{l}\text { Cumple } \\
\text { parcialmente con el } \\
\text { criterio enunciado }\end{array}$ & 5 & $\begin{array}{l}\text { Se establece, } \\
\text { se implementa y } \\
\text { no se mantiene }\end{array}$ \\
\hline C $\quad \begin{array}{l}\text { Cumple con el } \\
\text { mínimo del criterio } \\
\text { enunciado }\end{array}$ & 3 & $\begin{array}{l}\text { Se establece, } \\
\text { no se implementa, no } \\
\text { se mantiene }\end{array}$ \\
\hline D $\quad \begin{array}{l}\text { No cumple con el } \\
\text { criterio enunciado }\end{array}$ & 0 & $\begin{array}{l}\text { No se establece, no se } \\
\text { implementa, no se } \\
\text { mantiene }\end{array}$ \\
&
\end{tabular}

Tabla 3

Resultados del diagnóstico del SGC según NTP ISO 9001:2015

\begin{tabular}{lcl}
\hline \multicolumn{3}{c}{ RESULTADOS DE LA GESTIÓN EN CALIDAD DE LA FIMM } \\
\hline NUMERAL DE LA NORMA & $\begin{array}{c}\text { \% OBTENIDO DE } \\
\text { IMPLEMENTACION }\end{array}$ & $\begin{array}{l}\text { ACCIONES POR } \\
\text { REALIZAR }\end{array}$ \\
\hline $\begin{array}{l}\text { 4. CONTEXTO DE LA } \\
\text { ORGANIZACIÓN }\end{array}$ & $8 \%$ & IMPLEMENTAR \\
\hline $\begin{array}{l}\text { 5. LIDERAZGO } \\
\text { 6. PLANIFICACION }\end{array}$ & $30 \%$ & IMPLEMENTAR \\
\hline $\begin{array}{l}\text { 7. APOYO } \\
\text { 8. OPERACIÓN }\end{array}$ & $6 \%$ & IMPLEMENTAR \\
\hline $\begin{array}{l}\text { 9. EVALUACION DEL } \\
\text { DESEMPEÑO }\end{array}$ & $9 \%$ & IMPLEMENTAR \\
\hline 10. MEJORA & $0 \%$ & IMPLEMENTAR \\
\hline $\begin{array}{l}\text { TOTAL RESULTADO } \\
\text { IMPLEMENTACION }\end{array}$ & & IMPLMENTAR \\
\hline $\begin{array}{l}\text { Calificación global en la } \\
\text { Gestión de Calidad }\end{array}$ & & BAJO \\
\hline
\end{tabular}

\subsection{Diagnóstico de acreditación modelo SINEACE}

El diagnóstico describe la situación actual de la FIMM, asimismo, identifica oportunidades de mejora en relación al modelo de acreditación del SINEACE y su sistema organizativo actual contrastado con los estándares de acreditación según el SINEACE.

El diagnóstico fue resultado de entrevistas a los distintos integrantes de la FIMM, del mismo modo, la revisión de documentos y normas internas. La información procesada es presentada de dos maneras, una cualitativa, cuando muestra evidencia escrita y análisis documental y otra cuantitativa cuando evidencia una calificación de porcentaje de avance y cumplimiento del estándar (implementación). Tabla 4 
Tabla 4

Resultado del Diagnóstico del Modelo de Acreditación SINEACE

\begin{tabular}{|c|c|}
\hline \multicolumn{2}{|c|}{ RESULTADOS DE SINEACE } \\
\hline $\begin{array}{l}\text { DIMENSIÓN DEL MODELO DE } \\
\text { ACREDITACIÓN SINEACE }\end{array}$ & $\begin{array}{l}\text { \% OBTENIDO DE } \\
\text { IMPLEMENTACION }\end{array}$ \\
\hline 1. GESTIÓN ESTRATÉGICA & $6.67 \%$ \\
\hline 2. FORMACIÓN INTEGRAL & $11.54 \%$ \\
\hline 3. SOPORTE INSTITUCIONAL & $5.56 \%$ \\
\hline 4. RESULTADOS & $0.00 \%$ \\
\hline $\begin{array}{ll}\text { TOTAL } & \text { RESULTADO } \\
\text { IMPLEMENTACION } & \end{array}$ & $5.94 \%$ \\
\hline Calificación global en la Acreditación & BAJO \\
\hline
\end{tabular}

\subsection{Diagnóstico mediante el enfoque de sistema viable}

En forma integrada, la matriz de diagnóstico permite evidenciar las disfuncionalidades y deficiencias en los componentes organizacionales de la facultad.

A continuación los criterios de calificación utilizados en la "Matriz de diagnóstico de la cibernética organizacional y procesos". Tabla 5 y Tabla 6

\section{Tabla 5}

Criterios de calificación cibernética organizacional y procesos

\begin{tabular}{lcl}
\hline $\begin{array}{c}\text { Criterios de } \\
\text { Calificación }\end{array}$ & Puntaje & \multicolumn{1}{c}{ Estado } \\
\hline $\begin{array}{l}\text { En inicio o ausencia } \\
\text { total }\end{array}$ & 1 & $\begin{array}{l}\text { El componente o proceso no cumple con el } \\
\text { criterio de viabilidad. }\end{array}$ \\
\hline Operación básica & 2 & $\begin{array}{l}\text { El componente o proceso es ejecutado en } \\
\text { condiciones mínimas del criterio de viabilidad. }\end{array}$ \\
\hline En progreso & 3 & $\begin{array}{l}\text { El componente o proceso es ejecutado } \\
\text { considerando acciones en base al criterio de } \\
\text { viabilidad. }\end{array}$ \\
\hline Operativo & 4 & $\begin{array}{l}\text { El componente o proceso es ejecutado, } \\
\text { mantiene y toma acciones derivadas del criterio } \\
\text { de viabilidad. }\end{array}$ \\
\hline Realización plena & 5 & $\begin{array}{l}\text { El componente o proceso es ejecutado y afianza } \\
\text { el criterio de viabilidad. }\end{array}$ \\
\hline
\end{tabular}

Tabla 6

Matriz de diagnóstico de cibernética organizacional y procesos de la FIMM

\begin{tabular}{lcc}
\hline \multicolumn{1}{c}{ COMPONENTE } & $\begin{array}{c}\text { \% OBTENIDO DE } \\
\text { IMPLEMENTACION }\end{array}$ & $\begin{array}{c}\text { ACCIONES } \\
\text { POR } \\
\text { REALIZAR }\end{array}$ \\
\hline A. Conceptualización del sistema bajo estudio o focalizado & $20 \%$ & IMPLEMENTAR \\
\hline B. Definición de la organización & $29 \%$ & IMPLEMENTAR \\
\hline C. Autonomía & $27 \%$ & IMPLEMENTAR \\
\hline D. Balance interno & $20 \%$ & IMPLEMENTAR \\
\hline E. Inteligencia, adaptación y balance con el entorno & $33 \%$ & IMPLEMENTAR \\
\hline F. Mecanismo de identidad-política & $35 \%$ & IMPLEMENTAR \\
\hline$\quad$ TOTAL RESULTADO IMPLEMENTACION & \multicolumn{2}{c}{$\mathbf{2 7 \%}$} \\
\hline $\begin{array}{c}\text { Calificación global del nivel de viabilidad del sistema } \\
\text { organizativo }\end{array}$ & \multicolumn{2}{c}{ BAJO } \\
\hline
\end{tabular}

\section{Discusiones}

La situación de viabilidad de la organización está relacionado con la existencia de un conjunto de sistemas o funciones sistémicas nombradas por Beer como Sistema 1 (operación), Sistema 2 (coordinación), Sistema 3 (cohesión), Sistema $3 *$ (auditoría), Sistema 4 (adaptación) y Sistema 5 (identidad-política), así como un conjunto de relaciones entre estos sistemas funcionales y el entorno.
Aunado a ello, el estado de implementación del sistema de gestión de la calidad y la situación de cumplimiento del modelo SINEACE (Sistema Nacional de Evaluación y Acreditación de la Calidad Educativa). El diagnóstico organizacional implica evidenciar las obligaciones legales de la institución y los esfuerzos por mejorar la calidad de sus servicios y productos.

El estado de viabilidad de la organización expresa la situación de operatividad de los componentes de un sistema organizativo, es decir, ¿cómo es el funcionamiento de S1, $\mathrm{S} 2, \mathrm{~S} 3, \mathrm{~S} 3 *$, S4 y S5?; en las condiciones actuales y las patologías $\mathrm{y}$ arquetipos cibernéticos organizacionales acaecidos.

Las patologías organizacionales son estructurales y funcionales, las estructurales están "relacionadas con un inadecuado abordamiento de la complejidad total enfrentada por cualquier organización" [21] y las funcionales tratan de evidenciar si las cinco funciones (sistemas 1, 2, 3, 3*, 4, y 5) "necesarios para la viabilidad de la organización están convenientemente representados y funcionan adecuadamente" [21]. Ambas son útiles intelectuales que coadyuvan en el diagnóstico y diseño organizacional. Sin embargo, la comprensión de patologías en los integrantes de la organización es limitada por la poca difusión y mínimo esfuerzo de comprensión y aprendizaje. La tradición y costumbre de diagnosticar organizaciones mediante una perspectiva de organización funcional y jerárquica, en la mayoría de los casos es imperante.

Por su lado, los arquetipos cibernéticos organizacionales son de dos tipos, de identidad y estructurales. Los arquetipos de identidad revelan organizaciones que tienen transformaciones poco claras, las discrepancias entre el discurso utilizado y el comportamiento observado dificulta reconocer su identidad [22]. Los arquetipos estructurales revelan una utilización inadecuada de "recursos y una pobre regulación de las actividades organizacionales" [22]. No obstante, la descripción de los arquetipos consume dedicación durante el análisis y disponibilidad de participación de los integrantes de la organización.

El diagnóstico organizacional mediante la identificación y descripción de patologías estructurales y funcionales permite formular un plan de acción, de tratamiento con el propósito de cambiar y mejorar a la organización en forma integral. Del mismo modo, los arquetipos cibernéticos organizacionales coadyuvan en la intervención sistémica-cibernética de la organización. Aunque, a la fecha, la difusión y utilidad de las patologías y arquetipos están restringidas en algunas organizaciones y ámbitos académicos.

El sistema de gestión de la calidad es parte de un "sistema de gestión" de la organización relacionada con la calidad, un "sistema de gestión" es el "conjunto de elementos de una organización interrelacionados o que interactúan para establecer políticas, objetivos y procesos para lograr estos objetivos" [28]. Del mismo modo, "un 
sistema de gestión de la calidad en la organización aumenta la eficacia y eficiencia al cumplir los objetivos de la calidad de la organización, coadyuva a la mejora en la coordinación de los procesos de la organización, mejora en la comunicación entre los niveles y funciones de la organización, desarrollo y mejora de la capacidad de la organización y de sus integrantes para entregar los resultados deseados" [28]. Aunque, en la organización la implementación del sistema de gestión de la calidad incita a los integrantes a cumplir clausulas y requisitos de la norma, dejando de lado la mejora continua y el logro de los fines organizacionales.

El diagnóstico del nivel de implementación del sistema de gestión de la calidad permite evidenciar la situación actual de la organización y detalla la brecha de cumplimiento de los requisitos de la norma ISO 9001:2015. No obstante, en ningún caso proporciona las acciones para afrontar los efectos previstos y no previstos en la provisión de productos y servicios.

En el nivel de cumplimiento del modelo de acreditación del SINEACE, la acreditación es entendida como el "reconocimiento público al cumplimiento de estándares por parte de una institución, por sí misma no mejora la calidad. La estructura del modelo incluye cuatro categorías generales o dimensiones: una dimensión central de formación integral, una dimensión de gestión estratégica y una dimensión de soporte institucional; y la cuarta de resultados" [29]. El modelo y matriz de estándares de acreditación fue formulada con el propósito de reflexionar y promover un mayor análisis y valoración de la relación entre: ¿qué propone el programa de estudios?, ¿qué indudablemente realiza?, ¿qué consigue como resultado? y ¿qué tiene que hacer para mejorar?. Pretende ser una herramienta de gestión que fortalezca la autoevaluación, sitúe una práctica de mejora continua y lleve hacia la autorregulación. Sin embargo, en algunas oportunidades la institución de educación superior universitaria o programa de estudios, confunde el mejorar la situación actual a los fines de la educación superior con el simple cumplimiento de estándares en forma documental y menos de resultados.

El diagnóstico mediante la matriz del modelo de acreditación del SINEACE es un inicio formal y evidente para el logro de los estándares de calidad establecidos por el SINEACE en el modelo de acreditación respectivo. Asimismo, es la evidencia de evaluación orientada a la mejora de la calidad de la institución. Aunque, el diagnóstico mediante la matriz, en algunos casos, podría ser confundido por parte de los integrantes de la institución como la etapa de autoevaluación, no obstante, solo es un punto de inicio formal y consistente.

\section{Conclusiones}

- Las patologías, los arquetipos organizacionales y el MSV contribuyen en el diagnóstico organizacional, fortalecen los esfuerzos por determinar las disfuncionalidades y deficiencias en forma integral en la organización.

- Enfatizar el valor del diagnóstico organizacional mediante el enfoque sistémico y la cibernética organizacional, es reiterar la aplicación de herramientas que proporcionan, es decir, declarar la definición sistémica, mediciones de autorregulación, desdoblar la complejidad organizacional, diagnóstico de: autonomía y operación (S1), sincronización y estabilización (S2), cohesión ( $\mathrm{S} 3)$, auditoria y monitoreo $(\mathrm{S} 3 *)$, adaptación e inteligencia (S4), identidad y ethos (S5).

- La matriz de requisitos en base a la norma NTP ISO 9001:2015 evidencia el nivel de implementación del sistema de gestión de la calidad en la Facultad y coadyuva a determinar el plan de acción de mejora a emprender.

- La matriz del modelo de acreditación del SINEACE asiste en evidenciar el nivel de cumplimiento del estándar en la institución y es punto de inicio en la formulación de planes de mejora con fines de acreditación.

\section{AGRADECIMIENTOS}

A la Facultad de Ingeniería Metalúrgica y de Materiales por el desarrollo del proyecto de intervención sistémica cibernética.

\section{REFERENCIAS}

[1] SINEACE, Educación Superior en el Perú: Retos para el Aseguramiento de la Calidad, Lima: SINEACE, 2013.

[2] J. Rivera Palomino, "Ideele," 13 Diciembre 2018. [Online]. Available:

https://revistaideele.com/ideele/content/laproblem $\% \mathrm{C} 3 \%$ A 1 tica-universitaria-actual-y-unapropuesta-de-soluci\% $\%$ C3\%B3n.

[3] R. M. Taipe Castro, "Estudio sistémico interpretativo de una institución de educación superior universitaria para el diseño de un sistema de gestión," UNCP, Huancayo Perú, 2018.

[4] S. M. Flores and M. A. Ludueña, Teoría General de Sistemas y Cibernética, Cuaderno Nro 7, Buenos Aires: Grupo de Estudios de Sistemas Integrados, 1983.

[5] O. S. Donaires, M. G. Pinheiro, L. H. Cezarino and D. P. Martinelli, "Systemic model for diagnosis of the micro, small and medium enterprises from two cities from the countryside of the state of São Paulo in Brazil," Syst. Pract. Action Res., vol. 23, no. 3, pp. 221-236, 2010. 
[6] D. Cabrera, L. Cabrera, E. Powers, J. Solin and J. Kushner, "Applying systems thinking models of organizational design and change in community operational research," Eur. J. Oper. Res., vol. 268, no. 3, pp. 932-945, 2018.

[7] S. Luckett, S. Ngubane and B. Memela, "Designing a Management System for a Rural Community Development Organization Using a Systemic Action Research Process," Syst. Pract. Action Res., vol. 14, no. 4, pp. 517-542, 2001.

[8] S. Beer, "Recursion zero: Metamanagement," Syst. Pract., vol. 3, no. 3, pp. 315-326, 1990.

[9] A. Leonard, "The viable system model and its application to complex organizations," Syst. Pract. Action Res., vol. 22, no. 4, pp. 223-233, 2009.

[10] J. Pérez Ríos, Design and Diagnosis for Sustainable Organizations The Viable System Method, UK: Springer, 2012.

[11] S. Beer, Brain of the firm, Toronto: John Wiley \& Sons, 1981.

[12] B. Gould, "Organizations as systems: the viable systems model (VSM)," The Antidote, vol. 4, no. 1, pp. 31-36, 1999.

[13] M. Schwaninger, "Design for viable organizations: The diagnostic power of the viable system model," Kybernetes, vol. 35, no. 7-8, pp. 955-966, 2006.

[14] Z. Rezaee, A. Azar, A. M. B. Erz and D. Nayeri, "Application of Viable System Model in Diagnosis of Organizational Structure," Syst. Pract. Action Res., no. 2001,2018

[15] W. O'Grady, S. Morlidge and P. Rouse, "Evaluating the completeness and effectiveness of management control systems with cybernetic tools," Manag. Account. Res., vol. 33, pp. 1-15, 2016.

[16] J. Pérez Ríos, I. Velasco Jiménez and X. Lois Martínez Suárez, "Design and diagnosis for viable organizations in practice: University urban planning," Kybernetes, vol. 41, no. 3, pp. 291-317, 2012.

[17] N. Burgess and N. Wake, "The applicability of the Viable Systems Model as a diagnostic for small to medium sized enterprises," Int. J. Product. Perform. Manag., vol. 62, no. 1, pp. 29-46, 2013.

[18] M. Pfiffner, "Five experiences with the viable system model," Kybernetes, vol. 39, no. 9, pp. 1615-1626, 2010 .
[19] M. I. Tarride, R. A. Zamorano, S. N. Varela and J. González, "Healthy organizations: Toward a diagnostic method," Kybernetes, vol. 37, no. 8, pp. 1120-1150, 2008.

[20] P. F. Katina, "Metasystem pathologies (M-Path) method: phases and procedures," J. Manag. Dev., vol. 35 , no. 10 , pp. 1287-1301, 2016.

[21] J. Perez Ríos, "APLICACIÓN DE LA CIBERNÉTICA ORGANIZACIONAL AL ESTUDIO DE LA VIABILIDAD DE LAS ORGANIZACIONES. PATOLOGÍAS ORGANIZATIVAS FRECUENTES (PARTE $2^{\mathrm{a}} \mathrm{Y}$ FINAL)," DYNA, pp. 127-146, 2008.

[22] R. Espejo and A. Reyes, Organizational Systems: Managing Complexity with the Viable System Model, UK: Springer-Verlag Berlin Heidelberg, 2011.

[23] C. C. P. Pablo, "Identification of organisational pathologies: Exploration of social network analysis to support the viable system model diagnostic," Kybernetes, vol. 49, no. 2, pp. 285-312, 2019.

[24] S. Beer, Diagnosing the System for Organizations, London: Wiley, 1985.

[25] R. M. Taipe Castro, Identificación y análisis de la UNCP Tomo I, Huancayo-Perú: UNCP, 2009a.

[26] R. M. Taipe Castro, Diagnóstico de la universidad basado en el enfoque de sistema viable Tomo II, Huancayo-Perú: UNCP, 2009b.

[27] R. Taipe Castro, Diseño de la universidad basado en el enfoque de sistema viable. Tomo III, HuancayoPerú: UNCP, 2009c.

[28] ISO 2015, Norma Internacional ISO 9001, Geneva Suiza: ISO, 2015.

[29] SINEACE, Modelo de Acreditación para Programas de Estudios de Educación Superior Universitaria, Lima: SINEACE, 2016.

[30] Ministerio de Educación del Perú, Ley Universitaria $N^{\circ} 30220$, Lima, Lima: El Peruano, 2014.

[31] R. Fuenmayor, The Ontology and Epistemology of a Systems Approach: A Fundamental Study And an Application to the Phenomenon Development/Underdevelopment, Ph. D. Thesis, England: University of Lancaster, 1985. 
[32] R. Fuenmayor, El cultivo de la Verdad, Ibague: Ediciones Unibague, 2016.

[33] R. Fuenmayor, "The Roots of Reductionism: A Counter- Ontoepistemology for a Systems Approach," Systemic Practice and Action Research, Vol. 4, No. 5, pp. 419-449, 1991.

[34] R. M. Taipe Castro, "Diagnóstico y diseño organizacional de la Universidad Nacional del Centro del Perú," UNCP, Huancayo - Perú, 2008.

[35] R. M. Taipe Castro, Pensamiento sistémico en el sector público, Huancayo - Perú: UNCP, 2010.

[36] The Free Dictionary, "The Free Dictionary," 22 Agosto 2018. [Online]. Available: http://es.thefreedictionary.com/facultad.
[37] UNMSA, "Universidad Nacional Mayor de San Marcos," 24 Agosto 2018. [Online]. Available: http://www.unmsm.edu.pe/?url=inicio-historia.

[38] Universidad Autónoma de Barcelona, "MOOC UAB," 10 Enero 2018. [Online]. Available: http:/www.uab.cat/web/estudiar/mooc/-que-es-uncurso-mooc-1345668281247.html.

[39] Centro de Comunicación y Pedagogia, "Los MOOC: orígenes, historia y tipos," 16 Julio 2018. [Online]. Available: http://www.centrocp.com/los-moocorigenes-historia-y-tipos/. 